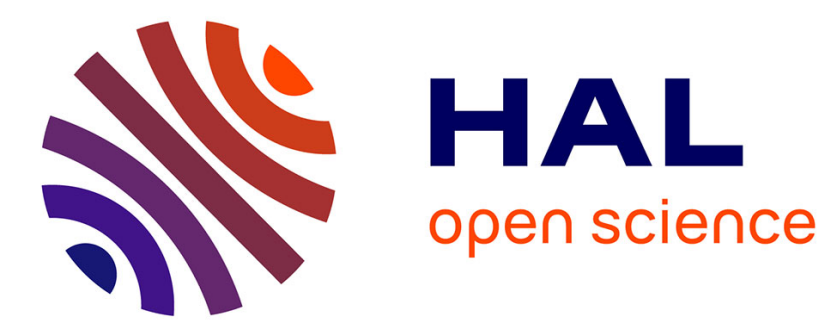

\title{
Rheological signature of microswimmer phase-locking under flow
}

Mohd Suhail Rizvi, Abdessamad Nait-Ouhra, Alexander Farutin, Philippe Peyla, Salima Rafaï, Chaouqi Misbah

\section{- To cite this version:}

Mohd Suhail Rizvi, Abdessamad Nait-Ouhra, Alexander Farutin, Philippe Peyla, Salima Rafaï, et al.. Rheological signature of microswimmer phase-locking under flow. Physical Review Fluids, 2019, 4 (10), 10.1103/PhysRevFluids.4.103302 . hal-02358198

\section{HAL Id: hal-02358198 \\ https://hal.science/hal-02358198}

Submitted on 19 Dec 2020

HAL is a multi-disciplinary open access archive for the deposit and dissemination of scientific research documents, whether they are published or not. The documents may come from teaching and research institutions in France or abroad, or from public or private research centers.
L'archive ouverte pluridisciplinaire HAL, est destinée au dépôt et à la diffusion de documents scientifiques de niveau recherche, publiés ou non, émanant des établissements d'enseignement et de recherche français ou étrangers, des laboratoires publics ou privés. 


\title{
Rheological signature of microswimmer phase-locking under flow
}

\author{
Mohd Suhail Rizvi, ${ }^{1, *}$ Abdessamad Nait-Ouhra, ${ }^{1,2}$ Alexander \\ Farutin, ${ }^{1}$ Philippe Peyla, ${ }^{1}$ Salima Rafai, ${ }^{1}$ and Chaouqi Misbah ${ }^{1, \dagger}$ \\ ${ }^{1}$ Laboratoire Interdisciplinaire de Physique, Université Grenoble Alpes and CNRS, F-38000 Grenoble, France \\ ${ }^{2}$ LaMCSCI, Faculty of Sciences, Mohammed V University of Rabat, 1014 Morocco
}

\begin{abstract}
Understanding macroscopic behaviors of suspensions of active particles is a challenging issue in the study of living fluids. We investigate the response of shape-change driven microswimmers to an external flow. In particular we estimate the viscosity of the dilute suspension of active microswimmers for the flow timescale close to that of the swimmer activity. We find that the shape activity leads either to an increase or decrease of the effective viscosity, depending on the shear rate. These opposite behaviors occurring for the same microswimmer originate from a phase-locking phenomenon between the swimmer shape oscillations and the applied flow. A simplified analytical model remarkably reproduces the results of full simulations, and offers a promising framework for the derivation of macroscopic constitutive laws for active matter.
\end{abstract}

*mohd-suhail.rizvi@univ-grenoble-alpes.fr

$\dagger$ chaouqi.misbah@univ-grenoble-alpes.fr 


\section{INTRODUCTION}

Because of the wide variety of the environments and ecosystems where micro-organisms live, their evolution has resulted in various ways of moving (gliding, twitching, swimming) towards their favorite chemical or food sources $[1,2]$. Examples of propulsion machinery include the cyclic beating of two flagella in a breast-stroke manner by $C$. reinhardtii [2], rotation of helical flagellar bundle by E. coli [1] or the repeated body-deformation of the Eutreptiella gymnastica [3]. Similarly, cells of the immune system develop ample shape changes to fight pathogens in complex environments, such as the extracellular matrix within tissues. There is now increasing evidence that cells of the immune system, among others, are able to swim in a fluid [4,5]. The micron size of cells and their low velocity ensure that inertia is negligible. Despite the diversity of micro-organisms, they all share a common feature, namely the repetitive and non-reciprocal temporal variation of forces on the fluid.

A popular swimmer model is the constant force-dipole which often discards the details of the active force generation machinery and retains the average behavior over one cycle of time-dependent active forces [6,7]. This swimmer can be categorized into two types- "puller" (eg. Chlamydomonas reinhardtii [7, 8]) and "pusher" (eg. E. coli [9]) depending on the sign of the dipole. A suspension of microswimmers displays unusual macroscopic rheology [8, 10-14]: a pushers' (pullers') suspension exhibits a lower (higher) effective viscosity than their respective passive counterparts. Furthermore, experimental studies have also shown a rich rheological behavior rarely recovered in theoretical models. For instance, a shear-thinning [8] (shear-thickening [13, 14]) nature of the pullers' (pushers') suspensions has been reported. So far, the main key ingredient of the models explaining the rheology of microswimmers (increase and decrease of viscosity due to activity), is rotational diffusion [15]. However, in general, microswimmers exhibit shape deformations (flagella motion, body shape changes, etc.) and their impact needs to be explored. It has been shown recently that the cyclic motion of flagella makes the cell follow very specific Jefferey's orbits that can affect rheology $[16]$.

In order to decipher the rheological behavior of swimmer suspensions, it seems relevant to consider the coupling between the flow and the time-dependent active forces. More generally, in most cases, shape changes of microswimmers are intrinsically related to propulsion mechanisms (flagellar beating or shape deformations). We show that shape change activity not only contributes to the viscosity of the suspension without evoking noise, but also uncovers new subtle effects. In particular, the active contribution to the viscosity is found to switch between positive and negative values depending on the magnitude of the applied shear flow for a given type (puller or pusher) of microswimmer. This results from a nonlinear coupling between the swimmer and the applied flow.

A widely adopted model for swimmers consists of a thin rod-like rigid shape with a given stresslet (force dipole), which is aligned with the rod and is either inward (puller) or outward (pusher). A deterministic rod under linear shear flow is known to exhibit tumbling (Jeffery orbit) and spends the same time in the upper and lower halves of the shear plane. As a consequence, no resulting active contribution to the viscosity is expected on average. Noise breaks this symmetry (the rod, then, spends more time in the upper half plane), giving rise to a net contribution of the active stress to the rheology $[6,12]$. Hence, in all available models, noise (due, for instance, to rotational diffusivity or tumbling of the microswimmers) is a crucial ingredient to recover a net effect of average active stresses on viscosity. Real swimmers undergo repeated shape changes, that lead to a non-trivial contribution to the active stress. Here we will address the effect of shape activity on the rheology of microswimmer suspensions in two cases: amoeboid swimmer and bead-spring microswimmer.

\section{RHEOLOGY OF MICROSWIMMER SUSPENSIONS}

\section{A. Amoeboid microswimmer}

We begin our study by considering an amoeboid microswimmer in 2d-space (Fig. 1) characterized by a fluid-filled impermeable membrane of perimeter $P_{0}$ and enclosed area $A_{0}$ immersed in another fluid [17, 18]. The swimmer has reduced area $\Gamma=4 \pi A_{0} / P_{0}^{2}$ (measuring deviation from a circle for which $\Gamma=1$ ) which allows the microswimmer deformation. $\eta$ and $\lambda \eta$ denote the respective viscosities of external and internal fluids. We consider the flow, in and outside of the swimmer to be in Stokes regime, following

$$
\nabla \cdot \mathbf{u}=0, \quad \eta_{i} \nabla^{2} \mathbf{u}(\mathbf{r}, t)=\nabla P(\mathbf{r}, t)
$$

where $\mathbf{u}$ and $P$ are the fluid velocity and pressure, respectively, and $\eta_{i}=\eta$ or $\lambda \eta$ depending on the location of $\mathbf{r}$. The shape of the swimmer at time $t$ is described by a closed curve $\mathbf{X}(s, t)$ in $x$ - $y$ plane, where $s$ is the curvilinear 


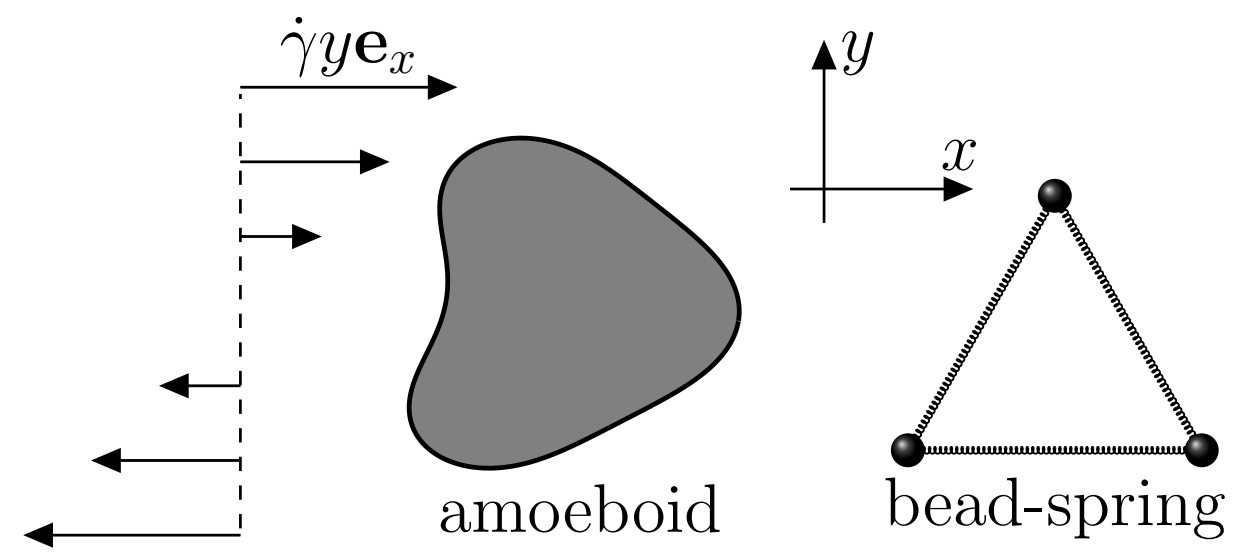

FIG. 1. Schematics of bead-spring and amoeboid microswimmers in shear flow.

coordinate along the membrane. We represent the membrane position $\mathbf{X}$ by a Fourier series

$$
\mathbf{X}(s)=\sum_{k=-k_{\max }}^{k_{\max }} X_{k} \exp (2 \pi i k s)
$$

where the complex amplitudes $X_{k}$ are the shape parameters of the membrane. Given the inextensibility of the membrane the application of active force results in generation of tension $\xi(s, t)$ which gives rise to a tangential force $\xi(s, t) \mathbf{t}(s, t)$. The resultant passive force density on the swimmer surface is, therefore, given by

$$
\mathbf{f}(s, t)=-\xi(s, t) c(s, t) \mathbf{n}(s, t)+\frac{d \xi(s, t)}{d s} \mathbf{t}(s, t)
$$

where $\mathbf{n}$ and $\mathbf{t}$ are the normal and tangential unit vectors in the membrane surface, respectively. In addition to the passive membrane forces, there is also an active force normal to the membrane which is also written in terms of Fourier harmonics as

$$
F_{a}(s, t)=\sum_{n=-n_{\max }, n \neq-1,0,1}^{n=n_{\max }} f_{n}(t) e^{i n s} .
$$

In the above, due to the fluid incompressibility the term corresponding to $n=0$, which corresponds to a constant pressure jump across the membrane, plays no role. For simplicity we set $f_{ \pm 1}(t)=0$ (we have kept the model as simple as possible by considering only the second and third harmonics). We require at least two harmonics to induce shape deformations which are not invariant under time-reversal a necessary condition for autonomous swimming (Scallop theorem [19]). We set $f_{2}=-f_{a} \cos \left(\omega_{a} t\right) / 2$ and $f_{3}=f_{a} \sin \left(\omega_{a} t\right) / 2$, where $\omega_{a}$ is the stroke frequency and $f_{a}$ is the active force amplitude. By considering only the real part of the Fourier harmonics expansion, we get the following active force

$$
F_{a}(s, t)=-f_{a} \cos \left(\omega_{a} t\right) \cos (2 s)+f_{a} \sin \left(\omega_{a} t\right) \cos (3 s)
$$

Including higher harmonics is not decisive for the swimming phenomenon [20]. Therefore, the total force density on the swimmer surface is given by

$$
\mathbf{F}(s, t)=F_{a}(s, t) \mathbf{n}+\mathbf{f}(s, t)+\mathbf{f}_{o}(t)+f_{t}(t) \mathbf{t}(s, t)
$$

where $\mathbf{f}_{o}(t)$ and $f_{t}(t) \mathbf{t}(s, t)$ allows to fulfill the force-free and torque-free conditions on the swimmer surface.

We define the non-dimensional active force amplitude $f=f_{a} /\left(\eta \omega_{a}\right)$ as the ratio between the time scales of the swimming strokes and fluid flow. This, together with hydrodynamic interactions, results in autonomous propulsion along a straight line $[17,20,21]$. We make use of Green's function techniques to write the membrane velocity as an integral equation expressing non-local hydrodynamics effects [22]. Details of the boundary integral method based numerical technique are given in the Appendix-A. 

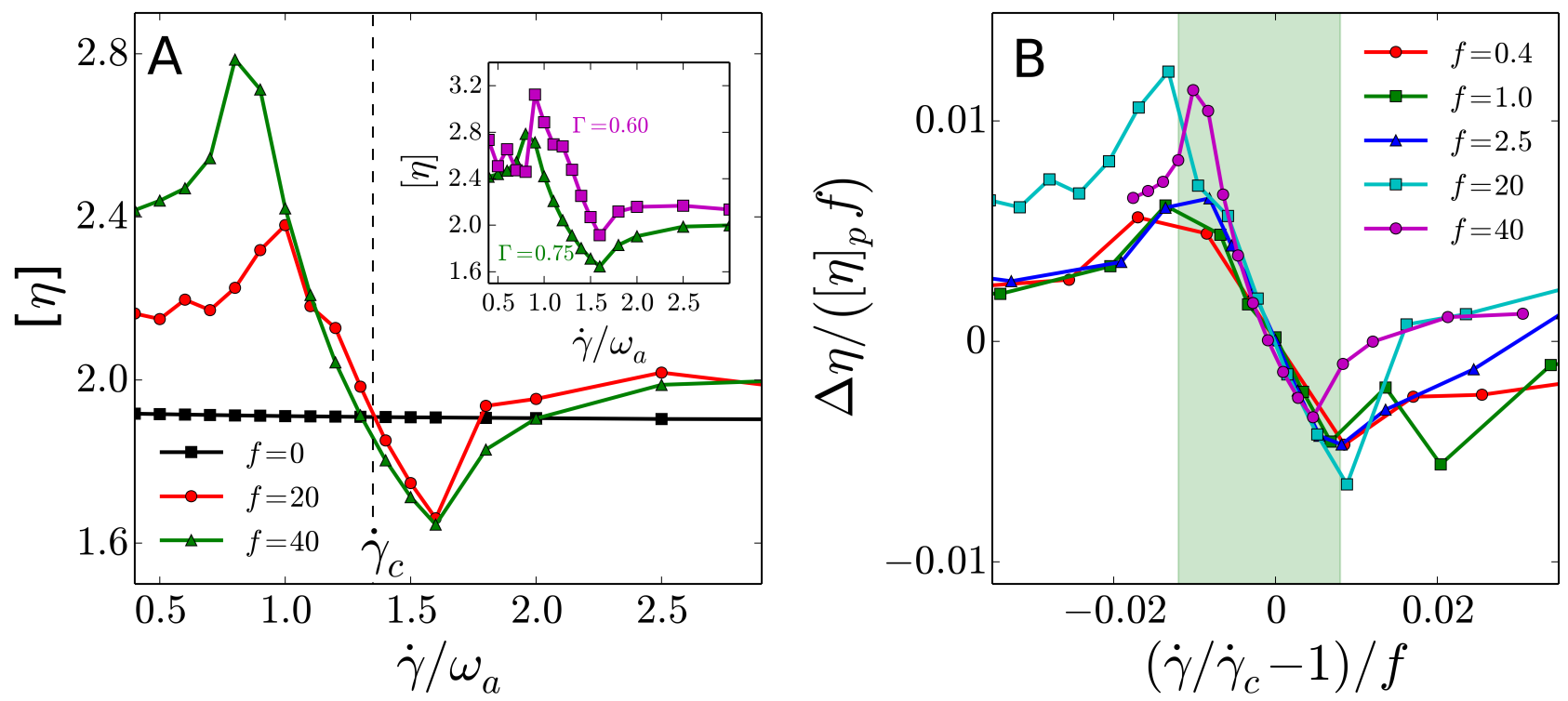

FIG. 2. (A) Intrinsic viscosities of passive vesicles $(f=0)$ and amoeboid microswimmers $(f>0)$ for $\Gamma=0.75$. $\dot{\gamma}_{c}$ marks the shear rate at which $\Delta \eta=0$. Inset shows $[\eta]$ for different $\Gamma$ with $f=40$. (B) For small $f$, the normalization of the shear rate difference $\dot{\gamma} / \dot{\gamma}_{c}-1$ and the active contribution to viscosity $\Delta \eta /[\eta]_{p}$ by $f$ results in a reasonable collapse of the curves, especially in the central region; $\lambda=20$.

The system is subjected to a linear shear flow of strength $\dot{\gamma}$ (Fig. 1) and the effective viscosity $\eta_{e f f}=\left\langle\sigma_{x y}\right\rangle / \dot{\gamma}$ is measured as the ratio of average shear stress (active+passive) and applied shear rate. For a suspension with swimmer volume fraction $\phi$, the intrinsic viscosity of the suspension $[\eta]=\left(\eta_{e f f}-\eta\right) / \phi \eta$ is obtained by the Batchelor's formula $[23]$ as

$$
[\eta]=\frac{1}{A_{0} \dot{\gamma} \eta}\left\langle-\int F_{x} y d s+\eta(\lambda-1) \int\left(u_{x} n_{y}+u_{y} n_{x}\right) d s\right\rangle
$$

where $x$ and $y$ subscripts denote the $x$ and $y$-components respectively, and $\mathbf{u}$ is the membrane velocity. Here the integral is performed over the membrane and $\langle\cdot\rangle$ refers to time average (to be specified later). We define the contribution of activity to viscosity as $\Delta \eta=[\eta]-[\eta]_{p}$ where $[\eta]_{p}$ is the intrinsic viscosity of the passive suspension, obtained by setting the active force to zero.

The dependence of intrinsic viscosity on the applied shear rates is summarized in Fig. 2. We observe that the contribution of activity switches from positive to negative at some critical shear rate $\dot{\gamma}_{c}$. Three successive regimes of $[\eta]$ are observed. For low values of shear rates $\left(\dot{\gamma}<\dot{\gamma}_{-}\right), d[\eta] / d \dot{\gamma}>0$ followed by $d[\eta] / d \dot{\gamma}<0$ for intermediate values of shear rates. Finally for high shear rates $\left(\dot{\gamma}>\dot{\gamma}_{+}\right),[\eta]$ again starts to increase with $\dot{\gamma}$. The transitions occur at shear rates $\dot{\gamma}_{\mp}$ which depend on the active force amplitude. A remarkable observation is that the normalized transition shear rates $\left(\dot{\gamma}_{\mp} / \dot{\gamma}_{c}-1\right) / f$ do not depend on the active force amplitudes $f$ (Fig. 2B) (see Sec.- III A for the motivation for this scaling.)

In $2 \mathrm{D}$ a dilute passive suspension of disks provides $[\eta]_{p}=2$ (equal to $5 / 2$ in $3 \mathrm{D}$ ) [24, 25]. For vesicles, it is about 1.8 for $\Gamma=0.75$ (Fig. 2A, black). In the presence of activity, the intrinsic viscosity attains values close to 3 (see Fig. 2A, green), showing the importance of activity (in the same proportion as found experimentally in 3D for Chlamydomonas [8]). From data shown in Fig. 2A (inset) obtained for two different deflation parameters $\Gamma$ we expect this value to be significantly larger than 3 for more deflated shapes such as that of Eutreptiella gymnastica. An estimate of active forces generated by the cells shows that the dimensionless force $f \gg 1$ (assuming $\eta \approx 1 \mathrm{cP}$ and $\omega_{a} \approx 1 \mathrm{~s}^{-1}$ )[26]. Owing to the membrane incompressibility $[\eta]$ saturates beyond a value of $f$ of few tens for $\Gamma=0.75$ (Fig. $2 \mathrm{~B}$ ). In order to gain insights into this rheological behavior, we estimated the effective viscosity of the dilute suspension of a much simpler system of active swimmer composed of beads and springs. 


\section{B. Bead-spring microswimmer}

We consider bead-spring microswimmer where three identical beads (radius $a$ each) are connected by linear springs (length $l \gg a$, stiffness $k=$ force/strain) in a triangular manner (Fig. 1) [27]. This is a variant of the model of flagellar swimming of $C$. reinhardtii proposed in $[28,29]$. In the Stokes regime, that is $R e=0$, we can write the equations of motion for each bead as

$$
\frac{d \mathbf{r}_{i}}{d t}=\mu \mathbf{f}_{i}(\mathbf{r}, t)+\sum_{j \neq i} \mathcal{G}_{i j} \cdot \mathbf{f}_{j}(\mathbf{r}, t)+\mathbf{u}^{\infty}
$$

where $\mu=(6 \pi \eta a)^{-1}$ is the bead mobility, $\mathbf{u}^{\infty}$ is the externally imposed flow and $\mathcal{G}_{i j}$ is the Oseen tensor

$$
\mathcal{G}_{i j}\left(\mathbf{r}_{i j}\right)=\frac{1}{8 \pi \eta}\left(\frac{\mathbf{I}}{r_{i j}}+\frac{\mathbf{r}_{i j} \otimes \mathbf{r}_{i j}}{r_{i j}^{3}}\right)
$$

describing the hydrodynamic interaction between the $i$ th and $j$ th beads, and $\mathbf{f}_{i}$ is the total force acting on $i$ th bead. By virtue of the hydrodynamic interactions among the beads, the active forces $f_{i j}=f_{a} \sin \left(\omega_{a} t+\alpha_{i j}\right)$ between $i$ th and $j$ th beads along the connecting spring lead to autonomous translation and/or rotation for appropriate values of $\alpha_{i j}$ [30]. In the absence of any external flow, for $\alpha_{12}=\alpha_{13}=0$ and $\alpha_{23}=\alpha \neq 0$ the swimmer performs translational motion. In general, the nonlinear nature of Eq. (8) makes it almost impossible to solve analytically. However, for $f_{a} \ll k$, the perturbation analysis around the equilibrium shape of the triangular microswimmer makes Eq. (8) analytically tractable. It has to be noted that the stresslet associated with this triangular microswimmer vanishes over the course of one cycle of active force. Therefore, it cannot be classified as puller or pusher microswimmer [27].

Under a linear shear flow, the contribution of the triangle to the suspension viscosity is given by the KramersKirkwood formula [31]

$$
[\eta]=-\frac{1}{v_{b} \dot{\gamma} \eta} \sum_{i}\left\langle y_{i}(t) f_{i}^{x}(t)\right\rangle
$$

where $v_{b}$ is the swimmer volume, $y_{i}(t)$ is the y-coordinate of the $i$ th bead and $f_{i}^{x}$ is the x-component of the total (active+passive) force acting on it. The resulting rheology is obtained numerically and shows the same qualitative features as in the case of amoeboid model (Fig. 3). To unravel the mechanisms that govern the observed transitions (from positive to negative active viscosity as a function of shear rate) we will, in the following, analytically quantify the coupling of the active forces and applied flow.

\section{ANALYTICAL CALCULATIONS}

\section{A. Triangle dynamics and phase locking}

For a passive vesicle or bead-spring triangle, one can describe the respective dynamics under shear flow in terms of the particle orientation $\varphi_{s}$ (see Appendix-C for details). Therefore, the time-averaging in Eqs. (7) and (10) for the passive particles can also be achieved by an averaging over $\varphi_{s}$. On the other hand, activity affects shape and thus the swimmer orientation. The phase related to activity is $\varphi_{a}=\omega_{a} t$, whereas the phase related to shear flow driven shape change is $\varphi_{s}=\omega_{s} t$ where, due to symmetry, the shape changes occur at a frequency twice of the tumbling frequency $\left(\omega_{s} \simeq \dot{\gamma}\right.$; as for the classical Jeffery orbit). This means that the shape configuration depends on both phases. For $\dot{\gamma} \ll \omega_{a}$ and $\dot{\gamma} \gg \omega_{a}$ (weak coupling), $\varphi_{s}$ can be considered independent of the activity and the time-averaging in Eqs. (7) and (10) has to be performed over the larger period $\left(4 \pi / \dot{\gamma}\right.$ or $\left.2 \pi / \omega_{a}\right)$. For $\dot{\gamma} \approx \omega_{a}$, however, there is a strong coupling between the swimmer orientation $\varphi_{s}$ and its activity $\varphi_{a}$. For weak deformation we have (which is an exact relation for rigid object)

$$
\frac{d \varphi_{s}}{d t} \approx \frac{1}{l^{2}}\left|\sum_{i=1}^{3} \mathbf{r}_{i}\left(\varphi_{s}, \varphi_{a}\right) \times \dot{\mathbf{r}}_{i}\left(\varphi_{s}, \varphi_{a}\right)\right|
$$

where $\mathbf{r}_{i}$ is the position vector of the $i$ th bead. The evolution equation of a bead position is expressed as a proportionality between $\dot{\mathbf{r}}_{i}\left(\varphi_{s}, \varphi_{a}\right)$ and the total force acting on the bead. The active part contains obviously $\varphi_{a}$ and the beads positions (via the spring force), which can be written as a function of $\varphi_{s}$ to leading order in deformation. Together 

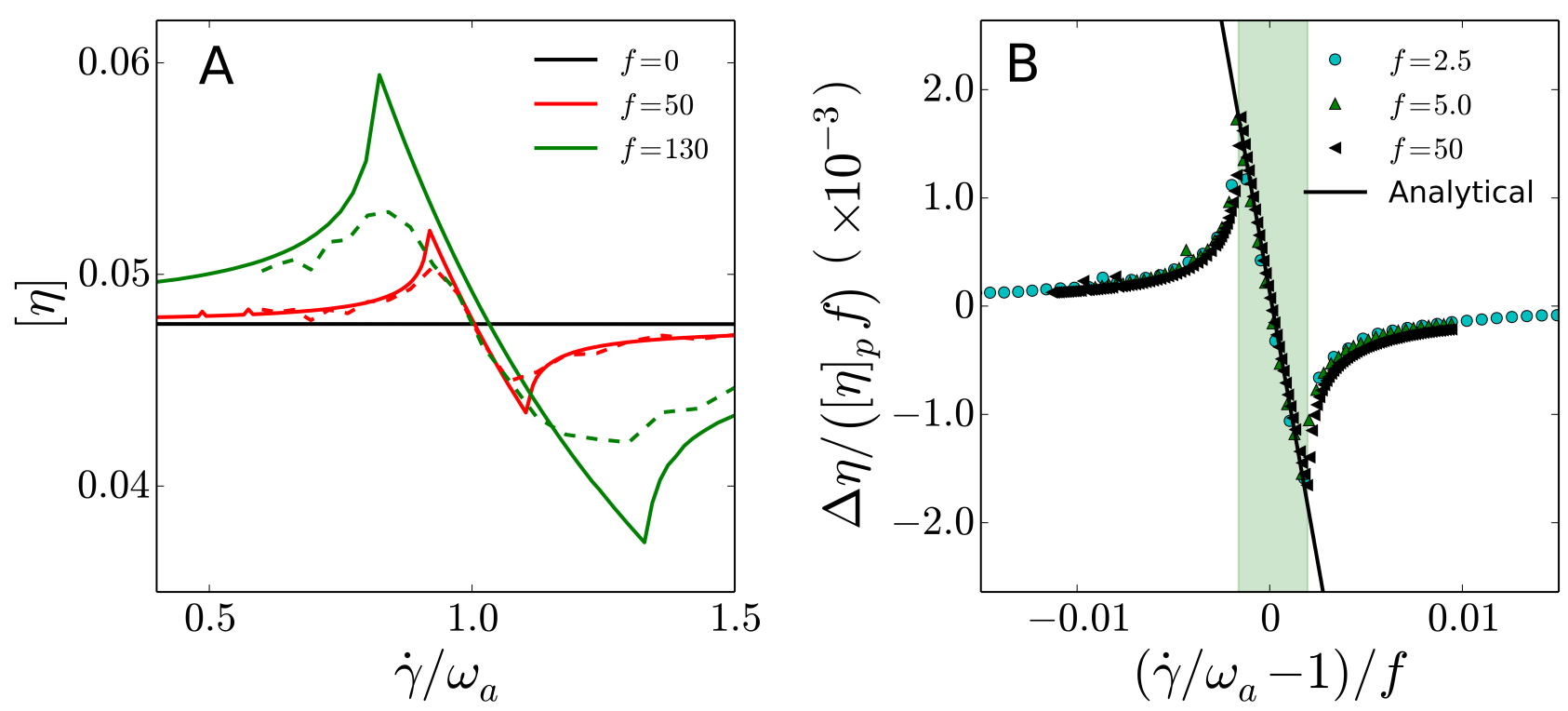

FIG. 3. (A) The intrinsic viscosities $\left(|\chi| / f_{a}=0,0.04\right.$ without (solid) and with (dashed) noise, respectively) and (B) normalized active viscosities, which follow Eq. (15) in the phase-locked regime, for the bead-spring triangles.

with definition (11) the time evolution of $\varphi_{s}$ is obtained (see Appendix-C.) If hydrodynamic interactions are ignored, along with the assumptions $f_{a} \ll k$ and $\dot{\gamma}, \omega_{a} \ll k \mu / l$, the phase equation assumes a simple analytical form, known as Adler equation [32]

$$
\frac{d \delta}{d t} \approx-\left(\dot{\gamma}-\omega_{a}\right)+A \dot{\gamma} \sin \left(\delta+\delta_{0}\right)
$$

where $\delta=\varphi_{s}-\varphi_{a}$,

$$
\cos \delta_{0}=\frac{3-3 \cos \alpha-2 \sin \alpha / \zeta}{\sqrt{2(1-\cos \alpha)\left(9+4 / \zeta^{2}\right)}}
$$

and

$$
A=2 \sqrt{2}\left(\frac{\sqrt{1-\cos \alpha}}{\sqrt{9+4 / \zeta^{2}}}\right) \frac{f_{a}}{k}
$$

depends on the magnitude of the triangle deformation $\left(f_{a} / k\right)$ due to the active force (see Appendix-B for more general form of the Adler equation for active triangle). In the above expressions, $\zeta=k \mu / \omega_{a} l$ is the ratio of the timescales associated with active force $\left(\sim 1 / \omega_{a}\right)$ and elastic deformation $(\sim l / k \mu)$.

Equation (12) is a well known equation in nonlinear systems and is used as a model for synchronization of oscillators $[33,34]$. It is apparent from the Eq. (12) that for $-A \leq\left(\dot{\gamma} / \omega_{a}-1\right) \leq A$ there is a "locking" of the two phases as $d \delta / d t=0$ and we obtain $\dot{\gamma}_{\mp}=\omega_{a}(1 \mp A)$ as the boundaries of the "phase-locked" regime (see Movies in [35]). For the amoeboid swimmer also we have the shear rates $\left(\gamma_{\mp}\right)$ proportional to the active force amplitude (Fig. 2B), a scaling inspired by Eq. (12). The phase locking phenomenon can be appreciated by analyzing the dependence of angular frequency of the shear flow driven triangle dynamics $\left(\omega_{s}\right)$ on the applied shear. By definition the angular frequency $\omega_{s}$ is twice the tumbling frequency due to the symmetry of the shear flow. Fig. (4)A shows that $\omega_{s}$ increases with the shear rate monotonically, except in the phase-locked regime where the synchronization of shear and activity modes results in $\omega_{s}=\omega_{a}$. The effect of phase locking is further reflected in the time dependence of the shear stress applied by the swimmer on fluid (Fig. 5). In the absence of activity under flow the particle has a cyclic motion with frequency $\omega_{s}$ (with the leading deformation mode behaving as $e^{i \omega_{s} t}+$ c.c.). The mode associated with activity is $e^{i \omega_{a} t}+$ c.c.. Due to nonlinear coupling, this generates new modes having frequencies $\omega_{s} \pm \omega_{a}$. In the phase-locked regime $\left(\omega_{s} \approx \omega_{a}\right)$ the system shows two frequencies: the basic one $\omega_{a}$ and $2 \omega_{a}$ (Fig. 5, middle panels). Outside the phase locked regime the system shows three disparate basic frequencies (generically, not rationally related to each other) $\omega_{a}$ and $\omega_{s} \pm \omega_{a}$, as seen in Fig. 5 (top and bottom panels). 

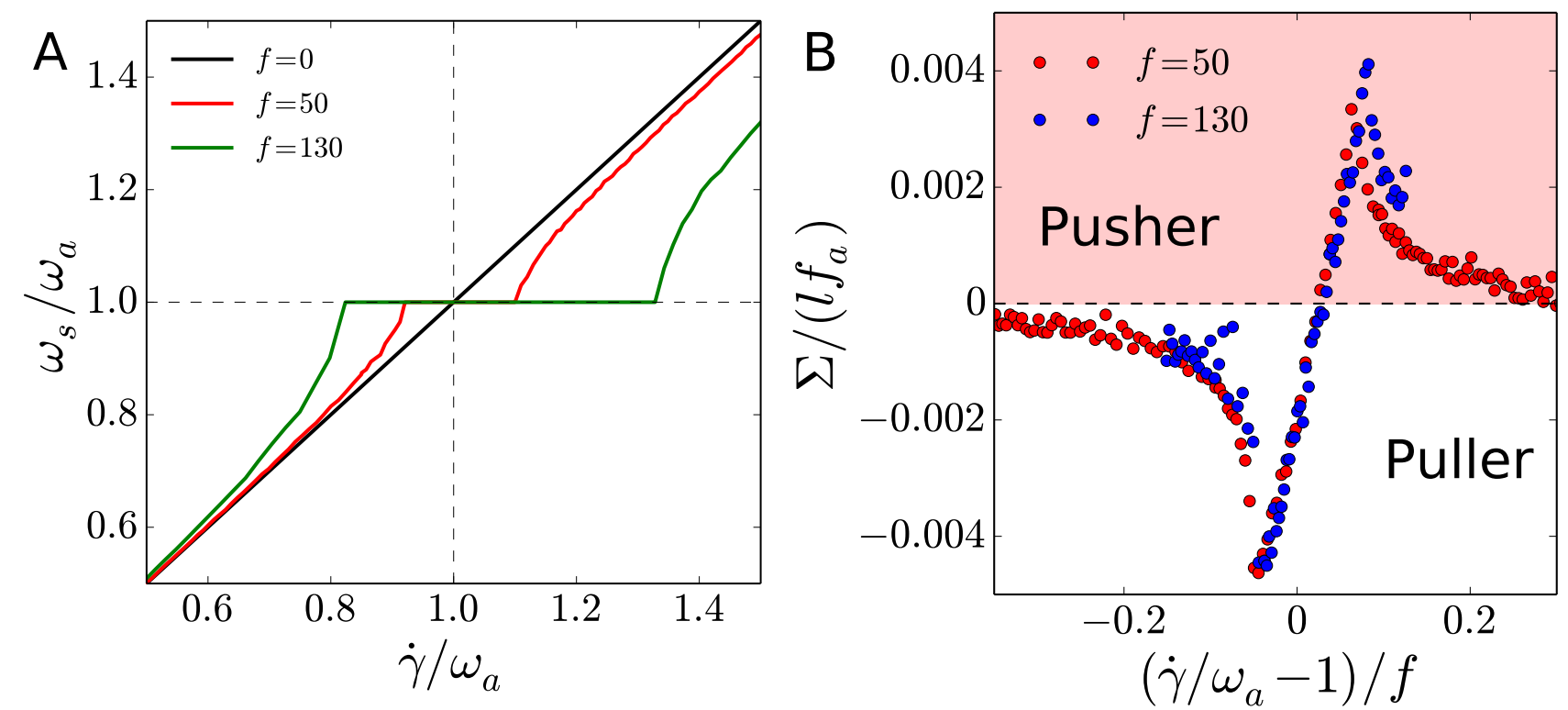

FIG. 4. Dependence of (A) $\omega_{s}$, the angular frequency of the shear flow driven dynamics and (B) the average stresslet $\Sigma$ for the bead-spring swimmer, as a function of applied shear rate, $\dot{\gamma}$
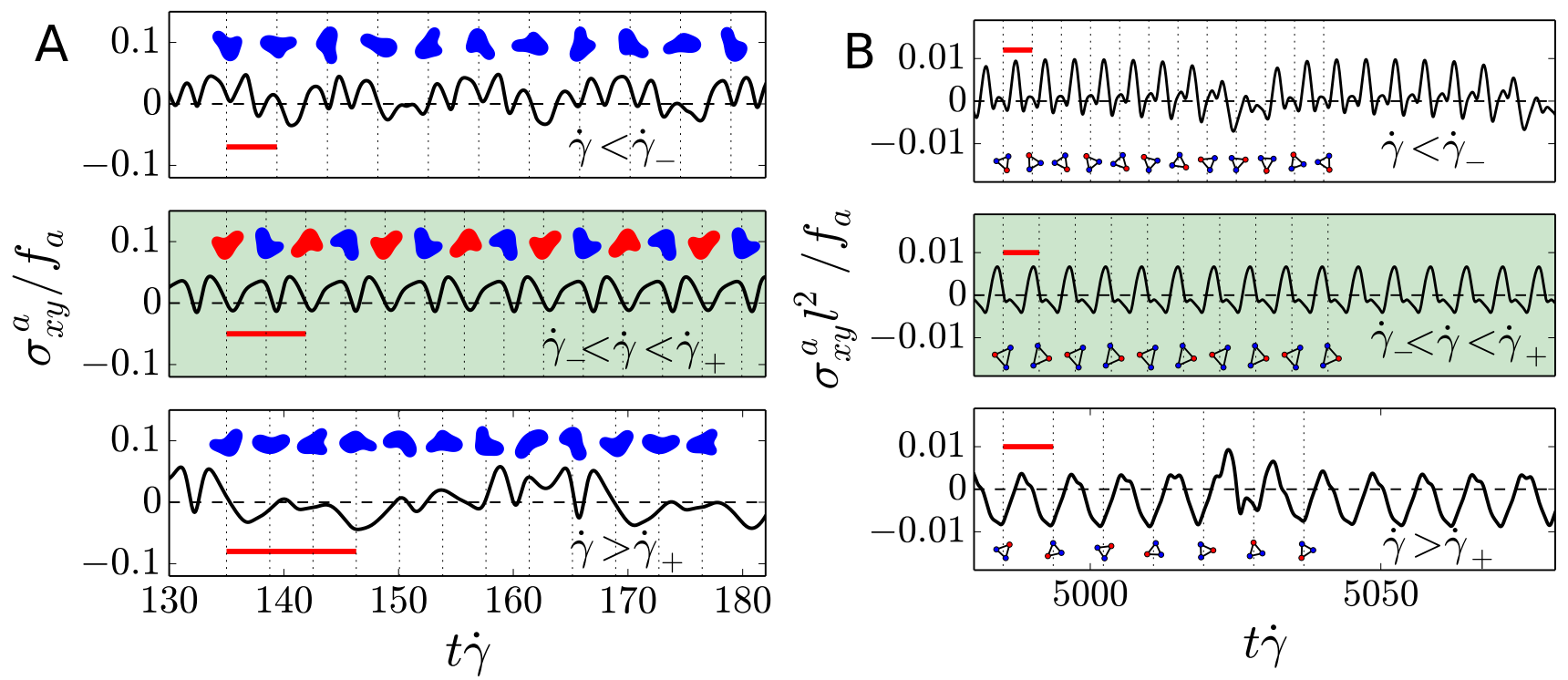

FIG. 5. Time dependence of the shear stress for (A) amoeboid and (B) bead-spring swimmers. Each panel also shows the evolution of swimmer conformations, showing a periodic character in the phase-locked regime (middle panels). The red bars designates the duration of the active force cycle.

\section{B. Effect of phase-locking on effective viscosity}

In the phase locked regime by using Eq. (10) we get

$$
\frac{\Delta \eta}{[\eta]_{p}} \approx \frac{[3 \zeta(\cos \alpha-\sin \alpha-1)+2(\cos \alpha+\sin \alpha-1)]}{\sqrt{\left(9 \zeta^{2}+4\right)(1-\cos \alpha)}}\left(\frac{\omega_{a}}{\dot{\gamma}}\right)^{2}\left(\frac{\dot{\gamma}}{\dot{\gamma}_{c}}-1\right)
$$


where

$$
\dot{\gamma}_{c}=\omega_{a}\left(1+3 \zeta^{2} \sqrt{\frac{2(1-\cos \alpha)}{9 \zeta^{2}+4}}\left(\frac{\cos \alpha+\sin \alpha-1}{3 \zeta(\cos \alpha-\sin \alpha-1)+2(\cos \alpha+\sin \alpha-1)}\right)\left(\frac{f_{a}}{k}\right)\right) .
$$

This shows that depending on $\alpha, \dot{\gamma}_{c}$ can increase or decrease with $f_{a}$. It is remarkable that $\Delta \eta /[\eta]_{p}$ is independent of $f_{a}$ in the phase-locked regime (Fig. 3B) which is also observed for amoeboid swimmer (Fig. 2B). The effect of active force amplitude $f_{a}$, however, does reflect in the maximum change in the viscosity, that is $\left|\Delta \eta /[\eta]_{p}\right|_{\max }$ which is obtained by substituting $\dot{\gamma}=\dot{\gamma}_{\mp}$ in Eq. (15). Since $\dot{\gamma}_{\mp}$ scale linearly with $f_{a}$ (at least for $f_{a} \ll k$ ) we have $\left|\Delta \eta /[\eta]_{p}\right|_{\max } \sim f_{a}$. It needs to be noted that this linear relation between maximum viscosity change and $f_{a}$ holds for $f_{a} \ll k$. For large $f_{a}$ the deformation of the triangle with linear springs can be very large and the results based on perturbation analysis may not hold.

Further, note that higher order phase-locking may also take place and Eq. (12) can be extended for $\delta_{m n}=m \varphi_{s}-n \varphi_{a}$ where $m \neq 0$ and $n \neq 0$ are arbitrary integers. The occurrence of higher order phase locking suggests multiple values of critical shear rates $\dot{\gamma}_{c}$ where transition between shear-thickening and shear-thinning behaviors can be observed (Fig. 6). A noteworthy point is that hydrodynamic interaction is not essential for the reported feature in Fig. 3A, albeit necessary for the self propulsion. The phase locking is found to be robust against several perturbations. For

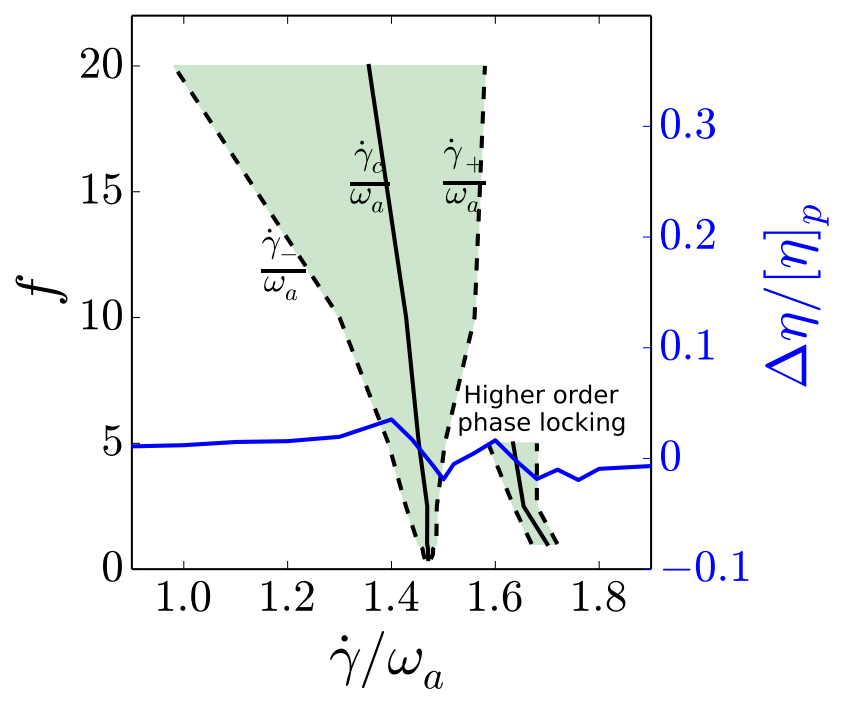

FIG. 6. Phase-locked regimes of the amoeboid swimmers for different $f . \Delta \eta /[\eta]_{p}$ for $f=5.0$ is shown as a reference.

the microscopic swimmers noise has a significant effect on their behavior. In order to study the influence of noise on the swimmer behavior under shear flow and the phase-locking phenomenon we consider the noise to be of two origins. First, the noise intrinsic to the swimmer coming from the mechanism of its activity. Its intrinsic nature ensures that it does not contribute to the total force and torque. Second, the noise experienced by the swimmer due to the fluctuations in the surrounding fluid flow. These fluctuations in fluid flow result in additional velocities to each bead of the triangular swimmer which we assume to be uncorrelated over time as well as among the beads. We consider the combined effect of noise in the form of random forces (proportional to the velocity fluctuations in the fluid) $\chi_{i}(t)$ on each bead such that they are uncorrelated, that is $\chi_{i}(t) \chi_{j}\left(t^{\prime}\right)=|\chi|^{2} \delta_{i j} \delta\left(t-t^{\prime}\right)$ where $|\chi|$ is the noise amplitude. This nature of the noise results in a nonzero contribution to the total force and torque to the swimmer, resulting in translational and rotational diffusion of the swimmer. We performed numerical simulations of the bead-spring swimmer with noise under shear flow using Euler-Maruyama method for the stochastic ordinary differential equations [36]. As shown in Fig. 3A the noise does not affect the qualitative features of the suspension viscosity as long as $|\chi| \ll f_{a}$. The consideration of noise reflects in the form of an additional diffusive term in the Adler equation (Eq. (27)) resulting in the diffusion of the phase-difference $\delta$ on a tilted washboard potential with occasional phase slips [34] as opposed to its deterministic behavior. 


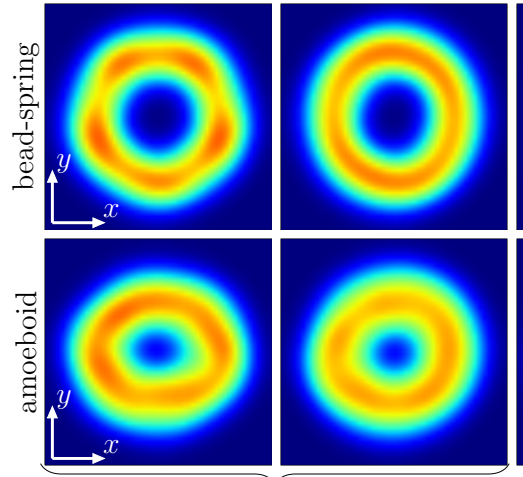

$d[\eta] / d \dot{\gamma}>0$
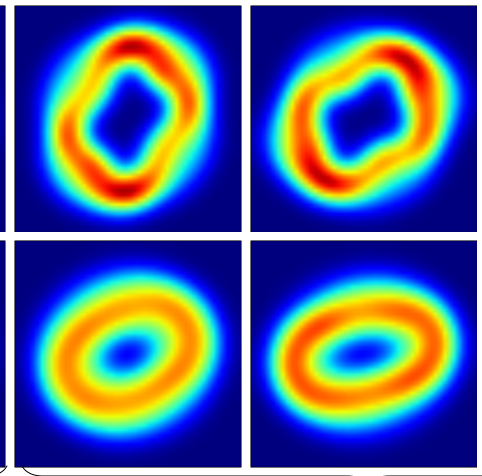

$d[\eta] / d \dot{\gamma}<0$ (phase-locked regime)
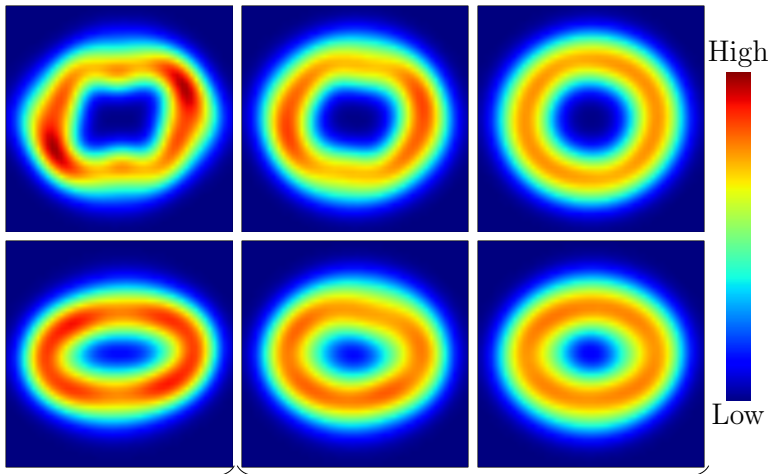

$d[\eta] / d \dot{\gamma}>0$

FIG. 7. Probability density of the presence of a bead (for triangle, upper panels) and swimmer membrane (for amoeboid, lower panels) under shear flow in the reference frame co-translating with the swimmer center.

\section{MECHANISMS LEADING TO THE VISCOSITY BEHAVIOR}

The microswimmers are usually categorized as puller or pusher depending on far-field velocity field generated by the swimmer. The leading order contribution in the far-field velocity field due to swimmer can be written in terms of the first moment of the force distribution, that is $\Sigma_{i j}=\sum_{i=1}^{3} \mathbf{f}_{i} \otimes \mathbf{r}_{j}$ for the bead-spring swimmer where $\mathbf{r}_{i}$ and $\mathbf{f}_{i}$ are the position and the total force on the $i$ th bead. In the absence of any external flow (such as shear flow in this paper), the symmetric nature of the swimmer results in the average velocity field over one active force cycle to be proportional to the average stresslet $\Sigma_{s}=\Sigma_{x x}-\Sigma_{y y}$ where $x$ is the direction of the swimmer propulsion. The puller and pusher nature of the swimmer is characterized by the sign of the stresslet $\Sigma_{s}\left(\Sigma_{s}<0\right.$ for puller and $\Sigma_{s}>0$ for pusher). The bead-spring triangular swimmer with three identical beads is known to demonstrate a puller and pusher characteristic within an active force cycle while on average its behavior is neutral $\left(\Sigma_{s}=0\right)$ [27]. In the presence of the shear flow the triangle demonstrates tumbling and we can define the stresslet in the reference frame attached to the swimmer as $\Sigma=\Sigma_{x^{\prime} x^{\prime}}-\Sigma_{y^{\prime} y^{\prime}}$ where the swimmer propulsion direction $x^{\prime}$ is co-rotating with it. We calculated the average value (over sufficiently long time to capture the effects of interaction between shear and activity modes) of stresslet $\Sigma$ of the bead-spring swimmer under different shear rates. Fig. 4B shows the transition of swimmer nature from pusher $(\Sigma<0)$ to puller $(\Sigma>0)$ at shear rate close to the angular frequency of active force. This transition between the two swimmer states coincides with the transition from $\Delta \eta>0$ to $\Delta \eta<0$. This demonstrates that for shape-deformation driven swimmers the puller-pusher nature is not an intrinsic property but depends on the external conditions, such as external flow (as shown here) or confinement [17].

In order to dig further into the origin of the viscosity change, we investigated the probability density of the microswimmer surface (or beads in case of bead-spring microswimmer) being at any location in the reference frame co-moving with the swimmer center of mass. This density represents the average microswimmer shape in the steady state (Fig. 7). For low $\dot{\gamma}$ the average shape first has an increasing cross section against the flow (meaning shear thickening), then a decrease of cross section until alignment with the flow in phase-locked regime, then an increase of cross section with $\dot{\gamma}$.

\section{DISCUSSION}

The phase locking is very generic due to nonlinear coupling of two oscillators. Shear flow can lead to tumbling (which is the first autonomous oscillator). This is the case, for example, for Chlamydomonas which undergoes (more or less complex) tumbling; see [8]. On the other hand the activity is materialized by a periodic beating of flagella (the second oscillator). The flow affects the motion of flagella, which in turns affects the tumbling dynamics, resulting in an effective coupling between the two oscillators (the activity mode and the tumbling one). We thus expect phase-locking to occur for various swimmers since tumbling and shape activity are quite common. Phase locking (at least the first order) seems to be absent for shakers (Eq. (27)). It needs to be pointed out that the mechanism of viscosity modulation by the swimmer activity shown here is fundamentally different from the previously known mechanisms which rely primarily on the rotational diffusion $[6,10,15]$ or an intrinsic tumbling mechanism $[12,15]$ of the microswimmer. In 
general, however, the two mechanisms- deterministic (shape activity as reported here) and stochastic (say diffusion or run-and-tumble) can be visible under different applied shear rates for the same microswimmer. For shear rates very different from that of the angular frequency $\omega_{a}$ of the swimming strokes it is the stochastic mechanism which has significant contribution as opposed to the shear rates close to $\omega_{a}$ where the deterministic mechanism takes over. Equation (15) shows that in the phase locked region (but for $\dot{\gamma} \neq \omega_{a}$ ) the active contribution to viscosity scales as $\Delta \eta /[\eta]_{p} \sim\left(\omega_{a} / \dot{\gamma}\right)^{2} \sim 1$. On the other hand for fixed shapes (slender body) with noise it is of order of $\Delta \eta /[\eta]_{p} \sim t_{r} / 15 t_{s}[15]$, where $t_{r}$ and $t_{s}$ are reorientation and swimming time (over swimmer size), respectively. For several swimmers $[8,13,15]$ the latter leads to $\Delta \eta /[\eta] \sim 1$, meaning that shape activity contribution may be of the same order as that of noise.

Since most of the experimental measurements of the active suspension viscosity has been for $\dot{\gamma} \ll \omega_{a}[8,13,14]$ the validation of the phenomenon reported in this paper would require further experimentation, especially for $\dot{\gamma} \approx \omega_{a}$. For flagellar microswimmers such as E. coli and C. reinhardtii $\omega_{a} \approx 100 \pi \mathrm{s}^{-1}$. Thus high enough shear rates are required for the phase-locking. In contrast, amoeboid swimmers $[3,4]$ are endowed with much longer stroke duration, approximately few seconds, requiring much lower shear rates. Finally, it will be an interesting task for future research to study multiple swimmers in order to analyze the interplay between their mutual coupling (which may trigger their synchronization) and the effect of external flow.

\section{APPENDIX}

\section{A. Numerical simulation of amoeboid microswimmer using Boundary Integral Method}

We consider a single swimmer in a linear shear flow

$$
\mathbf{u}^{\infty}=\dot{\gamma} y \hat{\mathbf{e}}_{x} .
$$

Considering very small Reynolds number, the flow can be regarded as a Stokes flow and we can use the boundary integral method (BIM) [22] to convert the Stokes equations into an integral form over the swimmer shape. The velocity at any point $\mathbf{r}$ in the domain can then be calculated by the boundary integral equation

$$
\begin{aligned}
\Lambda(\mathbf{r}) \mathbf{u}(\mathbf{r})= & \mathbf{u}^{\infty}(\mathbf{r})+\frac{1}{\eta} \oint \mathbf{F}\left(s^{\prime}\right) \cdot \mathcal{G}\left(\mathbf{X}\left(s^{\prime}\right), \mathbf{r}\right) d s^{\prime} \\
& +(1-\lambda) \oint \mathbf{u}\left(\mathbf{X}\left(s^{\prime}\right)\right) \cdot \mathcal{T}\left(\mathbf{X}\left(s^{\prime}\right), \mathbf{r}\right) \cdot \mathbf{n}\left(\mathbf{X}\left(s^{\prime}\right)\right) d s^{\prime}
\end{aligned}
$$

where $\mathcal{G}$ and $\mathcal{T}$ are the single and double layer Green's functions, respectively, and the integrals are performed over the swimmer surface. The term $\Lambda$ is defined as

$$
\Lambda(\mathbf{r})= \begin{cases}\lambda, & \text { if } \mathbf{r} \text { is inside the swimmer } \\ (1+\lambda) / 2, & \text { if } \mathbf{r} \text { is on a swimmer surface } \\ 1, & \text { if } \mathbf{r} \text { is outside of swimmer }\end{cases}
$$

Once the velocity on the membrane is known, the evolution of vesicle shape is obtained from a simple fixed time step Euler scheme

$$
X_{k}(t+\Delta t)=X_{k}(t)+u_{k}(t) \Delta t
$$

where $u_{k}(t)$ is obtained by taking Fourier transform of the velocity calculated from Eq. (18) at time $t$. Ideally, fluid incompressibility and membrane impermeability should automatically ensure the swimmer volume to be a constant but a drift due to numerical scheme can not be ruled out. To correct this drift, we inflate or deflate the swimmer through homogeneous normal deformation.

We performed several confirmatory simulations with refined meshes and time steps, sampling points and Fourier harmonics to ensure the numerical accuracy of the scheme. This verification led us to compromise between efficiency and accuracy and characterize each swimmer surface by 63 Fourier harmonics. Further, we used 2048 sampling points to resolve the short-range hydrodynamics interactions. We calculated the swimmer surface velocity at 128 sampling points. 


\section{B. Bead-spring microswimmer}

\section{Passive triangle in shear flow}

We can obtain the passive counterpart of the active bead-spring microswimmer by setting $f_{a}=0$. Once it is placed in shear flow $\dot{\gamma}$ it undergoes rotation and also oscillatory deformations. For $\dot{\gamma} \ll k \mu / l$ it is easy to see that the time-scale of triangle rotation and deformation is $4 \pi / \dot{\gamma}$. For small $\dot{\gamma}$ we can define the phase of the passive triangle $\varphi_{s}$ as the angle made by one the triangle beads with some pre-specified direction in the triangle plane. Therefore, all the properties of the passive triangle under shear can be written in terms of $\varphi_{s}$. We can write the contribution of the passive triangle to total shear stress in the form of Kramers-Kirkwood stress [31] as

$$
\sigma_{K K}^{x y}=\left\langle\sum_{i=1}^{3} f_{i}^{x}\left(\varphi_{s}\right) y_{i}\left(\varphi_{s}\right)\right\rangle
$$

where averaging is performed over $\varphi_{s} \in[0,2 \pi]$, that is all triangle configurations. For small triangle deformation, the force on any bead can be approximated as $f_{i}^{x, y}\left(\varphi_{s}\right)=\sum_{n=1}^{3} \sum_{j \neq i}\left(P_{i j}^{x, y} \sin \left(n \varphi_{s}\right)+Q_{i j}^{x, y} \cos \left(n \varphi_{s}\right)\right)$, where the $n \varphi_{s}$ dependence is due to the three-fold symmetric shape of the triangle. Similarly, the small deformation assumption also gives $y_{i}\left(\varphi_{s}\right)=R_{i} \sin \varphi_{s}+S_{i} \cos \varphi_{s}$. The coefficients $P_{i j}^{x, y}, Q_{i j}^{x, y}, R_{i}$ and $S_{i}$ are determined from the equations of motion of the beads (Eq. 8). Substituting the values of these coefficients in the Eq. (21) gives the effective viscosity of the passive bead-spring triangle suspension to be

$$
\eta_{p}=\eta+\frac{\phi}{v_{b}} \frac{\sigma_{K K}^{x y}}{\dot{\gamma}} \approx \eta+\frac{\phi}{v_{b}} \frac{9 l^{2}}{4 \mu}\left(\frac{1}{c^{2}+9}\right)
$$

where $v_{b}$ is the swimmer volume, $\phi$ is the swimmer volume fraction in the suspension and $c=\dot{\gamma} l / k \mu$ is the capillary number. This demonstrates a shear-thinning behavior of the passive triangle under shear flow.

\section{Active triangle in shear flow}

a. No coupling between the shear flow driven dynamics and activity: For the active triangle in shear flow if we do not consider any coupling between the shear flow and activity driven dynamics the triangle undergoes tumbling at a constant rate (due to shear flow) and deformation (due to activity as well as flow). In the absence of the coupling, the two modes- activity and shear flow are independent of each other and all dynamical variables can be written in terms of $\varphi_{s}=\dot{\gamma} t / 2$ and $\varphi_{a}=\omega_{a} t$. Therefore, we can follow the same steps as for the passive triangle above. However, there are additional terms due to the triangle activity in the expressions for the force on the bead and but with additional dependence on activity which gives $f_{i}^{x, y}\left(\varphi_{s}\right)=\sum_{j \neq i}\left[\left(P_{i j}^{a} \sin \left(\omega_{a} t\right)+Q_{i j}^{a} \cos \left(\omega_{a} t\right)\right) \sin \left(\varphi_{s}\right)+\left(\tilde{P}_{i j}^{a} \sin \left(\omega_{a} t\right)+\tilde{Q}_{i j}^{a} \sin \left(\omega_{a} t\right)\right) \cos \left(\varphi_{s}\right)\right]+$ passive force, where coefficients $P_{i j}^{a}, Q_{i j}^{a}, \tilde{P}_{i j}^{a}$ and $\tilde{Q}_{i j}^{a}$ can be obtained by solving the Eq. (8) for an isolated active triangle without any external shear flow. We substitute these values in Eq. (21) and take an average over one cycle of active force to obtain

$\eta_{a} \approx \eta_{p}-\frac{\phi}{v_{b}}\left(\frac{f_{a}}{k}\right)^{2} \frac{l^{2}}{2 \mu}\left(\frac{54(\cos (\alpha-\beta)+\cos \alpha+\cos \beta+3)+4 \sqrt{3}(9 c \zeta+c / \zeta)(\sin \beta-\sin \alpha+\sin (\alpha-\beta))+729 \zeta^{2}}{(9 \zeta+1 / \zeta)(9 \zeta+4 / \zeta)\left(9+c^{2}\right)}\right)$

to be the effective suspension viscosity in the absence of coupling between activity and shear flow driven dynamics. It can be seen that for purely translation microswimmer $(\beta=0)$, we get

$$
\eta_{a} \approx \eta_{p}-\frac{\phi}{v_{b}}\left(\frac{f_{a}}{k}\right)^{2} \frac{l^{2}}{2 \mu}\left(\frac{108(2+\cos \alpha)+729 \zeta^{2}}{(9 \zeta+1 / \zeta)(9 \zeta+4 / \zeta)\left(9+c^{2}\right)}\right)
$$

which has a monotonic dependence on $\dot{\gamma}$ contrary to the observation in Fig. 2. Therefore, the interaction between the two modes of triangle deformation cannot be ignored and the assumption of $\varphi_{s} \sim t$ breaks down.

b. With coupling between the shear flow driven dynamics and activity: In order to study the effect 
of coupling we first need to obtain the dependence of $\varphi_{s}$ on $t$. Under the assumption of small deformation of the triangle, we can write

$$
\frac{d \varphi_{s}}{d t} \approx \frac{1}{l^{2}} \sum_{i=1}^{3}\left(x_{i}\left(\varphi_{s}, \varphi_{a}\right) \frac{d y_{i}}{d t}\left(\varphi_{s}, \varphi_{a}\right)-y_{i}\left(\varphi_{s}, \varphi_{a}\right) \frac{d x_{i}}{d t}\left(\varphi_{s}, \varphi_{a}\right)\right)
$$

where $\left(x_{i}, y_{i}\right)$ are the coordinates of the $i^{t h}$ bead. In this condition we can take $x_{i}\left(\varphi_{s}(t), \varphi_{a}(t)\right)=R_{i}^{x} \sin \varphi_{s}(t)+$ $S_{i}^{x} \cos \varphi_{s}(t)+R_{i}^{a x} \sin \varphi_{a}(t)+S_{i}^{a x} \cos \varphi_{a}(t)$ and $y_{i}\left(\varphi_{s}(t), \varphi_{a}(t)\right)=R_{i}^{y} \sin \varphi_{s}(t)+S_{i}^{y} \cos \varphi_{s}(t)+R_{i}^{a y} \sin \varphi_{a}(t)+$ $S_{i}^{a y} \cos \varphi_{a}(t)$. Substituting these values in the following equation for the phase difference

$$
\frac{d \delta}{d t}=\frac{d \varphi_{s}}{d t}-\frac{d \varphi_{a}}{d t}
$$

gives us the time dependence of the $\varphi_{s}$ since the phase of the activity $\varphi_{a}=\omega_{a} t$ is already known. In a more general setup, we can also have the activity described not by explicit time dependence but triangle configuration. We do not consider those cases here. Assuming the change in the phase $\varphi_{s}$ to be slower than the swimmer activity, we can use method of averaging. After substituting the aforementioned expressions for $x_{i}$ and $y_{i}$ in Eq. (25) and taking average over one cycle of the active force, we obtain following Adler equation

$$
\frac{d \delta}{d t} \approx-\left(\dot{\gamma}-\omega_{a}\right)+\frac{f_{a}}{k} \frac{\dot{\gamma}}{9 \zeta+4 / \zeta}\left(A_{c} \cos \delta+A_{s} \sin \delta\right)
$$

with

$$
\begin{aligned}
& A_{c}=-2(2 \cos \alpha-3 \zeta \sin \alpha)+3 \sqrt{3} \zeta(\cos \beta-1)+2(\cos \beta+1)+\sin \beta(3 \zeta+2 \sqrt{3}) \\
& A_{s}=-2(2 \sin \alpha+3 \zeta \cos \alpha)+2 \sqrt{3} \zeta(\cos \beta-1)+3 \zeta(\cos \beta+1)+\sin \beta(2-3 \sqrt{3} \zeta) .
\end{aligned}
$$

This demonstrates that for different $\alpha$ and $\beta$ (which correspond to the distribution of the active force on the amoeboid swimmer surface) the phase-locking is observed under shear flow. The corresponding window of the the shear rates, however, is dependent on the nature of the active force distribution $(\alpha$ and $\beta$ ). It can be seen that for $\alpha=\beta=0$ (no propulsion) we get $A_{c}=A_{s}=0$, implying lack of phase-locking.

\section{ACKNOWLEDGEMENTS}

We thank CNES (Centre National d'Etudes Spatiales) and the French-German University Programme "Living Fluids" (Grant CFDA-Q1-14) for the financial support. The simulations were performed on the Cactus cluster of the CIMENT infrastructure supported by the Rhône-Alpes region (GRANT CPER07_13 CIRA).

[1] Eric Lauga and Thomas R Powers, "The hydrodynamics of swimming microorganisms," Reports on Progress in Physics 72, 096601 (2009).

[2] J. Elgeti, R. G. Winkler, and G. Gompper, "Physics of microswimmers-single particle motion and collective behavior: a review," Rep Prog Phys 78, 056601 (2015).

[3] J. Throndsen, "Flagellates of Norwegian coastal waters." Norwegian Journal of Botany 16, 161-216 (1969).

[4] N. P. Barry and M. S. Bretscher, "Dictyostelium amoebae and neutrophils can swim," Proc. Natl. Acad. Sci. U.S.A. 107, $11376-11380(2010)$.

[5] M. Bergert, A. Erzberger, R. A. Desai, I. M. Aspalter, A. C. Oates, G. Charras, G. Salbreux, and E. K. Paluch, "Force transmission during adhesion-independent migration," Nat. Cell Biol. 17, 524-529 (2015).

[6] D. Saintillan, "The dilute rheology of swimming suspensions: A simple kinetic model," Experimental Mechanics 50, 1275-1281 (2010).

[7] K. Drescher, R. E. Goldstein, N. Michel, M. Polin, and I. Tuval, "Direct measurement of the flow field around swimming microorganisms," Phys. Rev. Lett. 105, 168101 (2010).

[8] S. Rafai, L. Jibuti, and P. Peyla, "Effective viscosity of microswimmer suspensions," Phys. Rev. Lett. 104, 098102 (2010).

[9] K. Drescher, J. Dunkel, L. H. Cisneros, S. Ganguly, and R. E. Goldstein, "Fluid dynamics and noise in bacterial cell-cell and cell-surface scattering," Proc. Natl. Acad. Sci. U.S.A. 108, 10940-10945 (2011).

[10] Y. Hatwalne, S. Ramaswamy, M. Rao, and R. A. Simha, "Rheology of active-particle suspensions," Phys. Rev. Lett. 92, 118101 (2004). 
[11] D. Saintillan, "Extensional rheology of active suspensions," Phys Rev E Stat Nonlin Soft Matter Phys 81, 056307 (2010).

[12] S. C. Takatori and J. F. Brady, "Superfluid Behavior of Active Suspensions from Diffusive Stretching," Phys. Rev. Lett. 118, 018003 (2017).

[13] J. Gachelin, G. Mino, H. Berthet, A. Lindner, A. Rousselet, and E. Clement, "Non-Newtonian viscosity of Escherichia coli suspensions," Phys. Rev. Lett. 110, 268103 (2013).

[14] H. M. Lopez, J. Gachelin, C. Douarche, H. Auradou, and E. Clement, "Turning Bacteria Suspensions into Superfluids," Phys. Rev. Lett. 115, 028301 (2015).

[15] D. Saintillan, "Rheology of Active Fluids," Annual Review of Fluid Mechanics 50, 563-592 (2018).

[16] L. Jibuti, W. Zimmermann, S. Rafai, and P. Peyla, "Effective viscosity of a suspension of flagellar-beating microswimmers: Three-dimensional modeling," Phys Rev E 96, 052610 (2017).

[17] H. Wu, M. Thiebaud, W. F. Hu, A. Farutin, S. Rafai, M. C. Lai, P. Peyla, and C. Misbah, "Amoeboid motion in confined geometry," Phys Rev E Stat Nonlin Soft Matter Phys 92, 050701 (2015).

[18] H. Wu, A. Farutin, W. F. Hu, M. Thiebaud, S. Rafai, P. Peyla, M. C. Lai, and C. Misbah, "Amoeboid swimming in a channel," Soft Matter 12, 7470-7484 (2016).

[19] E. M. Purcell, "Life at low reynolds number," American Journal of Physics 45 (1977), 10.1119/1.10903.

[20] A. Farutin, S. Rafai, D. K. Dysthe, A. Duperray, P. Peyla, and C. Misbah, "Amoeboid swimming: a generic self-propulsion of cells in fluids by means of membrane deformations," Phys. Rev. Lett. 111, 228102 (2013).

[21] Q. Wang and H. G. Othmer, "Computational analysis of amoeboid swimming at low Reynolds number," J Math Biol 72, 1893-1926 (2016).

[22] C. Pozrikidis, Boundary Integral and Singularity Methods for Linearized Viscous Flow, 1st ed. (Cambridge University Press, 1992).

[23] G. K. Batchelor, "The stress system in a suspension of force-free particles," Journal of Fluid Mechanics 41, 545570 (1970).

[24] John F. Brady, "The einstein viscosity correction in n dimensions," International Journal of Multiphase Flow 10, 113 114 (1983).

[25] Vincent Doyeux, Stephane Priem, Levan Jibuti, Alexander Farutin, Mourad Ismail, and Philippe Peyla, "Effective viscosity of two-dimensional suspensions: Confinement effects," Phys. Rev. Fluids 1, 043301 (2016).

[26] J. Fouchard, C. Bimbard, N. Bufi, P. Durand-Smet, A. Proag, A. Richert, O. Cardoso, and A. Asnacios, "Three-dimensional cell body shape dictates the onset of traction force generation and growth of focal adhesions," Proc. Natl. Acad. Sci. U.S.A. 111, 13075-13080 (2014).

[27] M. S. Rizvi, A. Farutin, and C. Misbah, "Three-bead steering microswimmers," Physical Review E 97, 023102 (2018).

[28] Benjamin M. Friedrich and Frank Julicher, "Flagellar synchronization independent of hydrodynamic interactions," Physical Review Letters 109, 138102(5) (2012).

[29] Kyriacos C. Leptos, Kirsty Y. Wan, Marco Polin, Idan Tuval, Adriana I. Pesci, and Raymond E. Goldstein, "Antiphase synchronization in a flagellar-dominance mutant of chlamydomonas," Physical Review Letters 111, 158101 (2013).

[30] M. S. Rizvi, A. Farutin, and C. Misbah, "Three-bead steering microswimmers," Physical Review E 97, 023102 (2018).

[31] M. Doi and S. F. Edwards, The Theory of Polymer Dynamics (Oxford University Press, 1998).

[32] R. Adler, "A Study of Locking Phenomena in Oscillators," Proceedings of the IRE 34, 351-357 (1946).

[33] Yoshiki Kuramoto, "Self-entrainment of a population of coupled non-linear oscillators," in International Symposium on Mathematical Problems in Theoretical Physics, edited by Huzihiro Araki (Springer Berlin Heidelberg, Berlin, Heidelberg, 1975) pp. 420-422.

[34] A. Pikovsky, M. Rosenblum, and J. Kurths, Synchronization, A universal concept in nonlinear sciences, 1st ed. (Cambridge University Press, 2001).

[35] ", Supplementary movies showing the dynamics of amoeboid and bead-spring microswimmers for three shear rates $\left(\dot{\gamma}<\dot{\gamma}_{-}\right.$, $\dot{\gamma}_{-}<\dot{\gamma}<\dot{\gamma}_{+}$and $\left.\dot{\gamma}>\dot{\gamma}_{+}\right)$in the reference frame co-moving the with the swimmer center of mass..

[36] Peter E. Kloeden and Eckhard Platen, Numerical Solution of Stochastic Differential Equations (Springer Science and Business Media, 1992). 